\title{
Resultados del tratamiento de la artrosis glenohumeral primaria mediante hemiartroplastía
}

\author{
Results of the treatment of primary glenohumeral osteoarthritis through hemiarthroplasty \\ Benjumea-Carrasco A, * López-Fernández J, ${ }^{\ddagger}$ López-Mombiela F, ${ }^{\S}$ \\ Aburto-Bernardo M,§ Gallo-Del Valle EJ,* Álvarez-González JC ${ }^{\S}$ \\ Hospital General Universitario «Gregorio Marañón», Madrid, España.
}

RESUMEN. Introducción: La artrosis glenohumeral conlleva a una pérdida funcional y a una disminución de la calidad de vida de muchos pacientes. Actualmente, no existe consenso en cuanto al uso de la hemiartroplastía o la artroplastía total como tratamiento definitivo para este padecimiento. El objetivo es mostrar los resultados del tratamiento de la artrosis glenohumeral primaria mediante hemiartroplastía en nuestro servicio. Material y métodos: Revisamos 19 hemiartroplastías (14 pacientes) realizadas entre 2004 y 2013 en pacientes con artrosis glenohumeral primaria sin alteración morfológica glenoidea. Se recabaron los datos de ocho pacientes (11 hemiartroplastías), valorándose el estado funcional, el dolor y la realización de actividades de la vida diaria mediante las escalas Constant, ASES y DASH preoperatorias, a los cinco años de seguimiento mínimo (rango 5-11 años), así como las complicaciones presentadas. Se comparó el estado preoperatorio y al final del seguimiento. Resultados: Se encontró una mejoría estadísticamente significativa $(\mathrm{p}<0.05)$ en todos los parámetros analizados en el estudio. La media del dolor en la escala EVA disminuyó de 8.89 a 2.67. La escala ASES mejoró de 13.51 a 63.51 puntos de media. El Constant aumentó de 20.11 a 65.11 puntos. La puntuación media preoperatoria en la escala DASH fue de 73.68 y la postoperatoria de 28.30 puntos. La supervivencia media del implante fue de $81.82 \%$ a los siete años de seguimiento medio. Conclusión: Obtu-
ABSTRACT. Introduction: Glenohumeral osteoarthritis leads to a functional loss and a decrease in the quality of life of many patients that suffers it. Currently there is no agreement on the use of hemiarthroplasty or total arthroplasty as definitive treatment. The objective is to show the results of the treatment of primary glenohumeral osteoarthritis through hemiarthroplasty in our service. Material and methods: We reviewed 14 patients (19 hemiarthroplasties) performed between 2004 and 2013 in patients diagnosed with primary glenohumeral osteoarthritis without glenoid morphological anomaly. We managed to collect data from eight patients (11 hemiarthroplasties), assessing functional status, pain, and performing activities of daily living using the Constant, ASES and DASH scores preoperatively and at five years of minimum followup (range 5-11 years), as well as related complications. The preoperative status was compared to the end of the follow-up. Results: A significant improvement was found in all the parameters analyzed in the study $(p<0.05)$. The average pain in EVA score decreased from 8.89 to 2.67. The ASES improved from 13.51 to 63.51 half point. The Constant increase of 20.11 points to 65.11 points. The mean preoperative score in the DASH score was 73.68 and 28.30 postoperatively. The average survival of the implant was $81.82 \%$ in the seven years of average followup. Conclusion: Shoulder hemiarthroplasty obtains good

\section{Nivel de evidencia: IV}

* Servicio de Cirugía Ortopédica y Traumatología. Hospital General Universitario «Gregorio Marañón», Madrid, España.

* Servicio de Cirugía Ortopédica y Traumatología. Hospital Universitario «Infanta Leonor», Madrid, España.

§ Unidad de Cirugía de Hombro y Codo. Servicio de Cirugía Ortopédica y Traumatología. Hospital General Universitario «Gregorio Marañón», Madrid, España.

Dirección para correspondencia:

Antonio Benjumea-Carrasco

Servicio de Cirugía Ortopédica y Traumatología. Hospital General Universitario «Gregorio Marañón». Madrid, España.

Dirección institucional: Calle Doctor Esquerdo Núm. 46,

28007, Madrid (Madrid), España.

Teléfono: +34 676590341, Fax: 915866475

E-mail: antonio.benjumea.carrasco@gmail.com

https://dx.doi.org/10.35366/95317

doi: $10.35366 / 95317$ 
vimos buenos resultados funcionales con la artrosis glenohumeral primaria, presentándose pocas complicaciones. La causa del fracaso fue la erosión glenoidea.

Palabras clave: Hemiartroplastía hombro, artrosis glenohumeral primaria, resultados.

\section{Introducción}

La artrosis primaria de hombro, también conocida como artrosis glenohumeral o enfermedad articular degenerativa del hombro, supone un deterioro progresivo y gradual, tanto mecánico como biológico del cartílago y otros tejidos articulares, incluyendo los elementos óseos y la cápsula articular. ${ }^{1}$ La pérdida de función en el hombro conlleva limitaciones para realizar actividades cotidianas y laborales, así como a estados de ansiedad o depresión. ${ }^{2}$ Cuando el tratamiento conservador fracasa, la hemiartroplastía de hombro (HA) y la artroplastía total de hombro (AT) constituyen alternativas válidas. $^{3}$

Existe controversia sobre cuál de estas dos opciones es la más adecuada. Por una parte, la AT puede causar deterioro del stock óseo, enfermedad de partículas y aflojamiento del componente glenoideo; razones que constituyen motivos de dolor y pérdida de la función; ${ }^{4}$ por otra parte, la HA puede ocasionar erosión glenoidea, que es la principal causa de deterioro clínico y funcional en estos pacientes y que es causa de revisión a corto y mediano plazo. .,6,7 $^{5}$

El propósito de este estudio es analizar retrospectivamente los resultados obtenidos en el tratamiento de la artrosis glenohumeral primaria mediante hemiartroplastía en nuestro servicio.

\section{Material y métodos}

Se revisaron de manera retrospectiva, entre Junio de 2004 y Junio de 2013, a aquellos pacientes con artrosis glenohumeral primaria con afectación glenoidea limitada y a quienes se les hubiera realizado una HA en nuestro centro.

Los criterios de inclusión fueron: artrosis glenohumeral primaria con afectación glenoidea tipos A y B1 de la clasificación de Walch y tipos 0 y 1 de la clasificación de Habermeyer; ${ }^{8}$ edad superior a los 60 años con seguimiento mínimo de cinco años; manguito rotador competente y ausencia de inestabilidad glenohumeral.

Como criterios de exclusión, se descartaron aquéllos con diagnóstico de artrosis secundarias a artropatías inflamatorias, rotura masiva de manguito rotador y postraumáticas tras capsulorrafia o con antecedentes de infección. También, se eliminaron del análisis a aquellos pacientes en los que la documentación clínica no estaba completa.

Durante el periodo de tiempo mencionado se realizaron un total de 19 HA en 14 pacientes; asimismo, se revisaron sus historias clínicas, con la autorización del estudio por la functional results in the treatment of primary glenohumeral osteoarthritis with few complications. The cause of the failure were glenoid erosion in all the cases.

Keywords: Shoulder hemiarthroplasty, primary glenohumeral osteoarthritis, results.

Comisión de investigación del Instituto de Investigación Sanitaria «Gregorio Marañón». Todos los pacientes firmaron un consentimiento informado en el que aceptaron el uso de sus datos clínicos para ser incluidos en el estudio.

Del total de casos, no pudieron ser analizados seis pacientes (ocho hombros); tres pacientes (cinco hombros) fallecieron durante el periodo del estudio y otros tres (tres hemiartroplastía de hombro) se perdieron en el seguimiento. Los ocho pacientes (11 hombros) restantes intervenidos fueron incluidos en el estudio y analizados; de estos 11 casos, dos precisaron reintervención durante el seguimiento para la colocación del componente glenoideo, los cuales se excluyeron del análisis final (Figura 1).

Las características demográficas de los 11 hombros intervenidos son las siguientes: nueve de ellos $(81.81 \%)$ correspondieron a mujeres y dos (18.28\%) a varones; la edad media de la muestra fue de 72.82 años (62-82 años; DE: 5.91). Todos los pacientes eran diestros; se intervinieron seis hombros derechos (54.5\%) y cinco izquierdos (45.5\%). El seguimiento medio fue de 82.89 meses (61-131; DE: 24.56), considerándose éste como el tiempo desde la cirugía hasta el final del seguimiento.

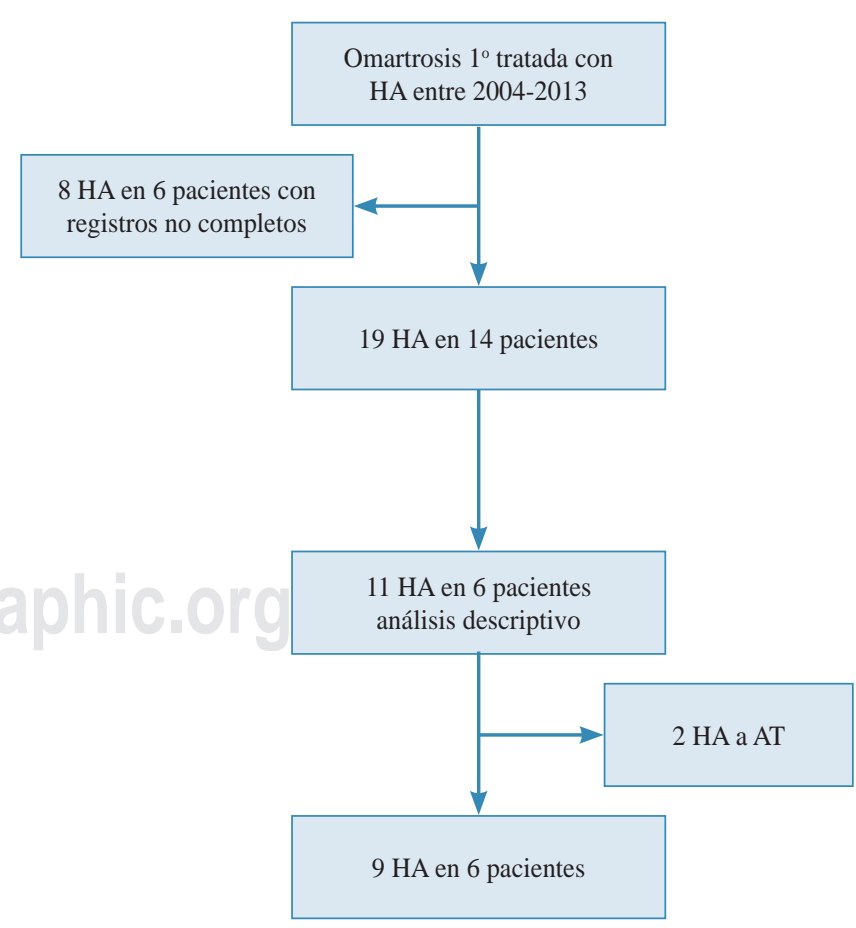

Figura 1: Diagrama de flujo de pacientes.

$\mathrm{HA}=$ Hemiartroplastía de hombro, AT = Artroplastía total de hombro. 

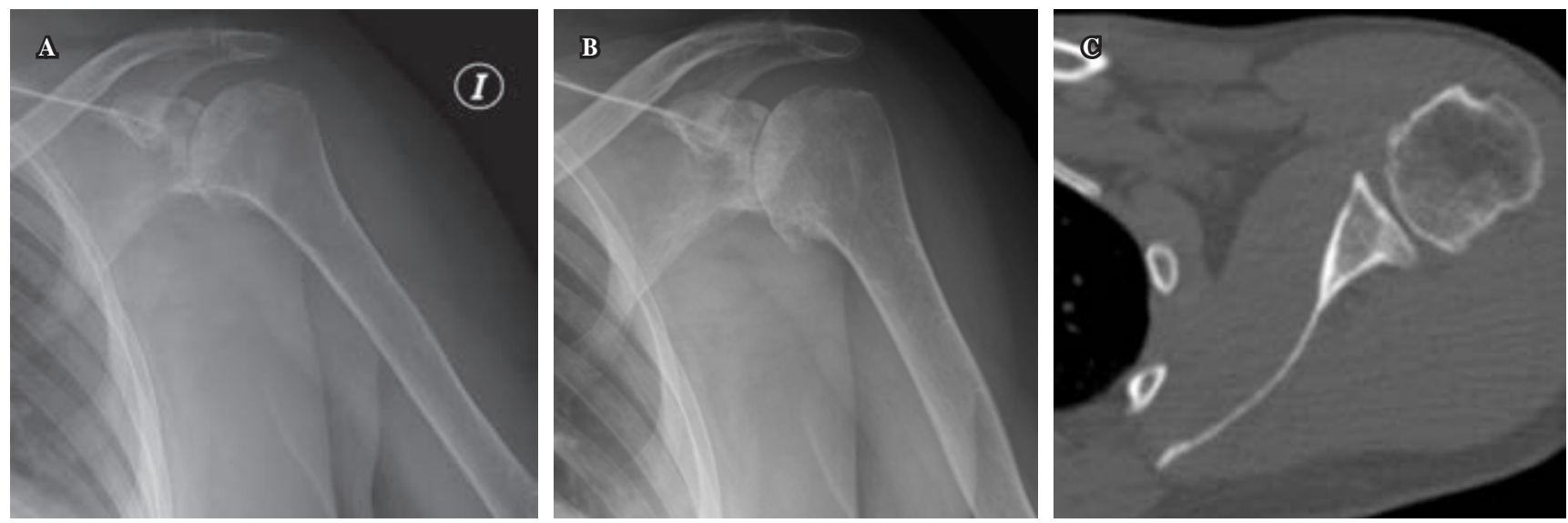

Figura 2: Imágenes preoperatorias de uno de los pacientes intervenidos. A) Proyección anteroposterior donde se objetiva esclerosis subcondral y disminución del espacio articular en la articulación glenohumeral izquierda. B) Proyección oblicua donde destaca el osteofito a nivel anteroinferior en la cabeza humeral. C) Corte axial del la TAC preoperatoria de este paciente.

Todos los pacientes disponían de estudios preoperatorios de imagen mediante radiografía convencional y tomografía axial computarizada (TAC) (Figura 2).

La cirugía se realizó mediante un abordaje deltopectoral, con tenodesis de la porción larga del bíceps a pectoral mayor y tenotomía del subescapular que se reparó al cierre. El modelo de prótesis empleado en todos los casos fue el GLOBAL (DePuy Warsaw, $\operatorname{In}^{\circledR}$ ).

La técnica quirúrgica siguió las recomendaciones de la técnica original. Para ello, se usaron implantes no cementados, con retroversión de $30^{\circ}$, empleando cabezas humerales excéntricas cuando se adaptaban mejor a la osteotomía humeral. En el postoperatorio inmediato, se colocó un cabestrillo en el miembro afectado, comenzando con ejercicios de codo y pendulares de hombro tan pronto la situación clínica del paciente lo permitiera. El alta hospitalaria se produjo en todos los casos entre el tercer y quinto día postoperatorio. Entre el alta y el inicio de la rehabilitación asistida a las cuatro semanas de la intervención, se instruyó a los pacientes en la realización de ejercicios de Codman.

Los pacientes fueron revisados a las seis semanas, tres meses, seis meses y al año. A partir de ese momento, se realizaron controles bianuales hasta el final del seguimiento. En el postoperatorio inmediato, así como en cada visita, se realizó un estudio radiográfico en dos proyecciones: anteroposterior y lateral en plano escapular.

La valoración funcional se realizó con las escalas: ASES, ${ }^{9}$ la cual evalúa la calidad de vida en pacientes con hombro doloroso; Constant, ${ }^{10}$ que valora aspectos tanto objetivos, como la movilidad articular y la fuerza, así como subjetivos, como dolor y actividades de la vida diaria y la escala DASH, ${ }^{11}$ que es un instrumento específico de medición de la calidad de vida relacionada con los problemas del miembro superior.

El estado preoperatorio de la glenoides se evaluó a través de las clasificaciones de Walch y Habermeyer, ${ }^{8,12}$ mediante imágenes de TAC, obteniendo los siguientes datos: con respecto a la forma glenoidea en el plano axial, $77.8 \%$ de los pacientes pertenecía al grupo A2 de la clasificación de Walch, mientras que $22.2 \%$ fue clasificado como B1; en relación con el plano coronal, según la escala de Habermeyer, 77.8\% presentó un tipo 0 y 22.2\% un tipo 1.

\section{Análisis estadístico}

El análisis estadístico se realizó mediante el programa SPSS V11.0. Se empleó el test de Wilcoxon para el análisis de datos apareados. Se compararon los datos de las escalas ASES, Constant y DASH, así como el dolor, el balance articular y la fuerza. Se buscó si existían diferencias preoperatorias en las variables analizadas respecto al final del seguimiento.

Los autores del presente estudio declaran no haber tenido ninguna fuente de financiación para la realización del mismo.

\begin{tabular}{|c|c|c|c|}
\hline & Preoperatorio & $\begin{array}{l}\text { Final del } \\
\text { seguimiento }\end{array}$ & $\mathrm{P}$ \\
\hline EVA (dolor) & 8.89 & 2.67 (10) & 0.007 \\
\hline ASES & 13.51 & 63.51 & 0.008 \\
\hline Constante & 15.98 & 65.11 & 0.008 \\
\hline Dolor & 2.55 & 13.56 (15) & 0.011 \\
\hline Actividad & 5.56 & $15.98(20)$ & 0.008 \\
\hline Balance articular & 7.11 & $23.11(40)$ & 0.008 \\
\hline Fuerza & 4.89 & 13.89 (25) & 0.008 \\
\hline DASH & 73.68 & 28.30 & 0.008 \\
\hline
\end{tabular}




\begin{tabular}{|c|c|c|}
\hline \multicolumn{3}{|c|}{$\begin{array}{l}\text { Tabla 2: Se muestra el resultado desglosado } \\
\text { de cada ítem de las escala de ASES. }\end{array}$} \\
\hline & Preoperatorio & Postoperatorio \\
\hline Dolor & $9 / 9$ & $4 / 9$ \\
\hline Dolor nocturno & $9 / 9$ & $2 / 9$ \\
\hline $\begin{array}{l}\text { Uso de medicación } \\
\text { analgésica }\end{array}$ & $7 / 9$ & $2 / 9$ \\
\hline Uso de opioides & $4 / 9$ & $0 / 9$ \\
\hline $\begin{array}{l}\text { Puntuación media de } \\
\text { la EVA (sobre 10) }\end{array}$ & $8.89( \pm 0.93)$ & $2.67( \pm 2.06)$ \\
\hline Sensación de inestabilidad & $0 / 9$ & $0 / 9$ \\
\hline $\begin{array}{l}\text { Puntuación media de actividad } \\
\text { de la vida diaria (sobre 30) }\end{array}$ & $4.78( \pm 3.63)$ & $15.78( \pm 5.31)$ \\
\hline Puntuación total de ASES & $13.51( \pm 9.26)$ & $63.52( \pm 17.49)$ \\
\hline
\end{tabular}

\section{Resultados}

\section{Análisis descriptivo}

Se logró una mejoría postoperatoria en todos los parámetros revisados. La media del dolor en la puntuación de la EVA (escala visual análoga) disminuyó de 8.89 a 2.67. La puntuación total en la escala ASES mejoró 50 puntos de término medio. El Constant aumentó más de 40 puntos al final del seguimiento respecto al inicio de éste. La escala DASH disminuyó de 73.68 a 28.30 puntos. Los resultados se muestran en las Tablas 1 y 2.

En cuanto al análisis realizado para valorar si existían diferencias entre el estado preoperatorio y postoperatorio en las puntuaciones de las diferentes escalas, en todos los parámetros analizados se hallaron diferencias estadísticamente significativas. Los resultados se muestran en la Tabla 1.

Durante el seguimiento, se encontraron complicaciones en dos de los 11 pacientes (18.18\%). Ambos presentaron dolor, limitación funcional en el hombro intervenido y la radiografías mostraron ser compatibles con usura de glenoideas, precisando protetización de la misma a los 44 meses y a los 51 meses desde la fecha de la cirugía. La evolución posterior en estos dos pacientes ha resultado satisfactoria hasta el momento de finalizar este estudio.

No se tiene constancia de complicaciones mecánicas (inestabilidad o aflojamiento del componente humeral) ni infecciosas en las prótesis implantadas (Figura 3). Tampoco encontramos datos de pérdida de función a causa de desfuncionalización del manguito rotador que hayan precisado cirugía de revisión a la fecha de finalización del presente estudio.

\section{Discusión}

El tratamiento de la omartrosis mediante el empleo HA o AT continúa generando controversia. Hoy en día, existe un consenso respecto a emplear HA en casos seleccionados, por ejemplo, en déficit de stock óseo glenoideo y mor- fología no alterada de la glena. ${ }^{13}$ Los enfermos tratados mediante HA tienen el riesgo de presentar dolor relacionado con la erosión de la glenoides con el paso de los años. La AT tiene como principal complicación el aflojamiento del componente glenoideo a medio y largo plazo. ${ }^{7}$ Nuestros resultados muestran que, en el caso de la artrosis glenohumeral primaria en pacientes sin alteración en la morfología glenoidea, el tratamiento mediante HA consigue a largo plazo un buen resultado en cuanto al alivio de dolor, mejoría del balance articular y capacidad para desarrollar actividades cotidianas.

Los resultados obtenidos en nuestro trabajo nos muestran una mejoría del dolor que se mantiene a lo largo del tiempo, de modo similar a los resultados reportados en otras series. ${ }^{13,14,15}$ En 100\% de nuestros pacientes se presentaba dolor preoperatorio; más de 50\% continuaba sin dolor después de siete años de la intervención; de aquellos pacientes que manifestaron tener dolor, tan sólo un paciente refirió tener un dolor de intensidad moderada, el cual interfería de manera esporádica para algunas actividades.

Garstman y colaboradores ${ }^{13}$ en su estudio muestran una diferencia total de 54 puntos en las prótesis anatómicas y de 42 puntos en las HA al comparar el ASES preoperatorio con el postoperatorio. Además, reconocen que no hay diferencias estadísticamente significativas al comparar HA con AT en cuanto a la función, el rango articular, la fuerza ni la puntuación total de la escala ASES, aunque sí reconocen que las puntuaciones en AT son algo superiores. En nuestro trabajo, se aprecia una diferencia de 50 puntos entre el ASES prequirúrgico con el postquirúrgico, lo que es similar a lo encontrado por este autor para sus AT. Levine y su equipo de trabajo ${ }^{16}$ hallaron 68 puntos en la escala ASES postopera-

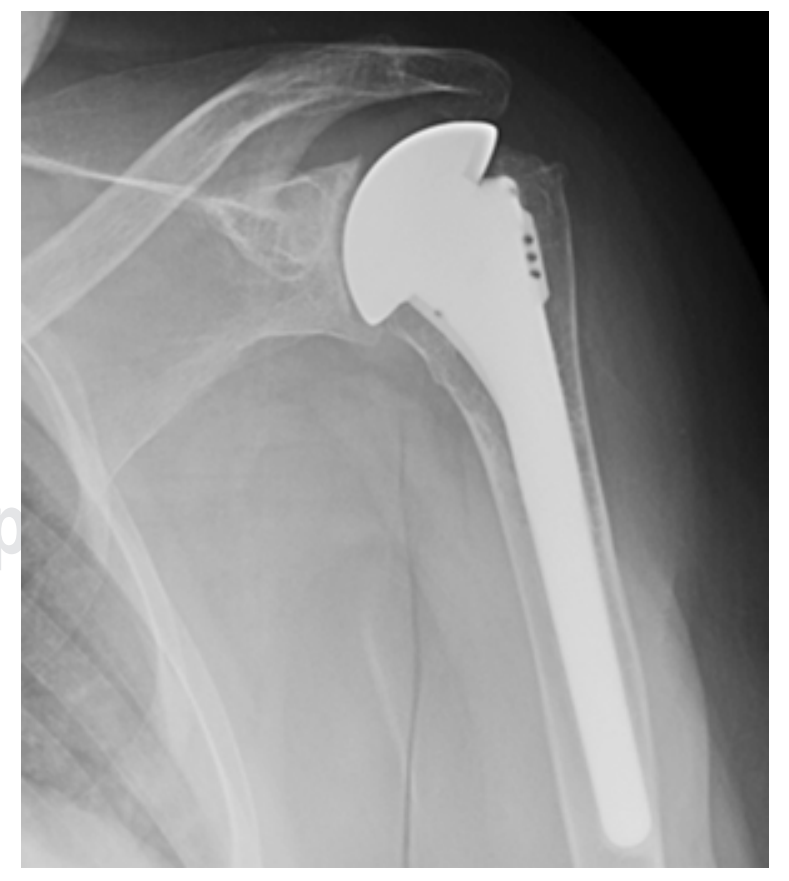

Figura 3: Radiografía AP al final del seguimiento. 
toria; esto es similar a nuestro trabajo. Estos autores refieren que en pacientes sin alteraciones en la glenoides presentan resultados satisfactorios con HA. Además, recalcan en su estudio que, a nivel global, los pacientes presentan mejores resultados cuando no tienen alteraciones morfológicas en la glenoides.

En cuanto a la escala Constant, en nuestro trabajo presentamos ganancia de 45 puntos. De manera similar, Gadea y su grupo de estudio ${ }^{14}$ presentaron una mejoría a los ocho años de seguimiento de 33.7 puntos, lo que determinaron como un excelente resultado funcional en su serie. Similar a nuestros hallazgos, Gartsman y colaboradores ${ }^{13}$ encontraron una mejoría global significativa en la movilidad articular a los tres años de media de seguimiento. Parece que esta mejoría se mantiene a lo largo del tiempo, de modo similar a lo encontrado en nuestro estudio. Al comparar los diferentes parámetros de la escala Constant en la bibliografía, vemos diferentes resultados. Tanto Gartsman como $\mathrm{Lo}^{13,15}$ no aprecian diferencias entre AT y HA en cuanto al balance articular a medio plazo en artrosis primaria. Edwards, ${ }^{17}$ por su parte, nos muestra que al analizar ganancias absolutas, la AT es superior a la HA en movilidad, actividad y resultado total de la escala, pero no encuentra superioridad de la AT en cuanto a la mejoría del dolor y fuerza. Todos estos autores concluyen que la HA es una opción válida para mejorar las actividades y el dolor en artrosis primaria.

Al valorar la calidad de vida, un metaanálisis de $2013^{18}$ no encontró diferencias en cuanto a la calidad de vida en los pacientes entre HA y AT. Nosotros empleamos la escala DASH como método para valorar de forma indirecta la calidad de vida y el desempeño de los pacientes de las actividades diarias. En nuestro estudio, se aprecia una mejora significativa en la puntuación DASH a los 10 años de seguimiento medio.

La necesidad de revisión por afectación de la glenoides es algo ampliamente descrito en la literatura. ${ }^{7,16}$ La supervivencia del implante en nuestra serie es de $81.82 \%$ a los siete años de seguimiento medio de nuestros pacientes, lo que es similar a lo mostrado en otro trabajo ${ }^{19}$ para pacientes por encima de los 60 años. En los dos casos que requirieron cirugía de revisión para protetización glenoidea, la causa fue el dolor provocado por la erosión glenoidea y el tiempo de aparición fue entre el tercer y quinto año postoperatorio; la revisión se llevó a cabo sin complicaciones reseñables.

En general, aunque la artroplastía total de hombro muestre valores superiores en cuanto a movilidad y resultado final de varias escalas, no queda demostrado que éstos sean superiores en cuanto al alivio de dolor y mejora en la calidad de vida respecto de la HA. Lo IK y otros,,$^{15}$ en un estudio prospectivo aleatorizado con un seguimiento medio de 4.8 años, no encontraron diferencias en los resultados clínicos entre ambas técnicas, pero sí una mayor tasa de complicaciones en las AT. Al contrario, Edwards ${ }^{17}$ no encontró diferencias significativas en la tasa de revisión en un estudio multicéntrico con una muestra de 690 hombros. Otros autores han publicado mejores resultados tras AT que tras HA en artrosis glenohumeral. ${ }^{20,21}$ Así, un metaanálisis realizado en $2005^{19}$ mostró una superioridad de la AT respecto a la HA, aunque los autores refieren que existe heterogeneidad en los datos, por lo que dicho análisis no es concluyente. Por todo lo anteriormente mencionado, consideramos que la decisión de elegir uno u otro procedimiento en estos pacientes continúa siendo controvertida.

Pese a ello, nuestro estudio presenta limitaciones. En primer lugar, el tamaño de la muestra es pequeño, aunque hay que matizar esto, ya que nuestro trabajo sólo incluye enfermedad articular primaria, sin lesión importante glenoidea en el momento de la intervención. La mayoría de las publicaciones revisadas por los autores del presente estudio incluyen artrosis de diversa etiología, lo que da pie a que se presenten muestras mayores y son, en muchos casos, multicéntricos, lo que permite reunir más casos, pero también a expensas de aumentar la variabilidad. En segundo lugar, el análisis se ve afectado por una pérdida de $40 \%$ de los casos intervenidos. Esto es debido, al menos en parte, al periodo de inclusión del estudio, que recoge casos durante 14 años y como límite de 60 años para incluirlos.

Como conclusión, defendemos que el objetivo principal a la hora de plantear un tratamiento quirúrgico mediante artroplastía de sustitución en un paciente con artrosis primaria sin alteraciones morfológicas glenoideas es aliviar el dolor, mejorar la función y conservar la calidad de vida de estos enfermos.

Consideramos que mediante la hemiartroplastía se consiguen todos estos objetivos y no existe necesidad de realizar artroplastías totales que incrementen la complejidad técnica, tiempo quirúrgico y costo, considerando el porcentaje de pacientes en los que ha sido necesario cirugía de revisión, similar al de otras publicaciones, tal como hemos expuesto antes y que, precisamente, esto se ha resuelto sin complicaciones relevantes.

Bibliografía

1. Millett PJ, Gobezie R, Boykin RE. Shoulder osteoarthritis: diagnosis and management. Am Fam Physician. 2008; 78(5): 605-11.

2. Memel DS, Kirwan JR, Sharp DJ, Hehir M. General practitioners miss disability and anxiety as well as depression in their patients with osteoarthritis. Br J Gen Pract. 2000; 50(457): 645-8.

3. Carter MJ, Mikuls TR, Nayak S, Fehringer EV, Michaud K. Impact of total shoulder arthroplasty on generic and shoulder-specific healthrelated quality-of-life measures: a systematic literature review and meta-analysis. J Bone Joint Surg Am. 2012; 94(17): e1271-9.

4. Matsen FA, Clinton J, Lynch J, Bertelsen A, Richardson ML. Glenoid component failure in total shoulder arthroplasty. J Bone Joint Surg Am. 2008; 90(4): 885-96.

5. Haines JF. The results of arthroplasty in osteoarthritis of the shoulder. J Bone Joint Surg Br. 2006; 88-B(4): 496-501.

6. Sandow M, Gartsman GKS. Total or hemiarthroplasty for OA shoulder meta-analysis of trials. J Bone JointSurg Br. 2002; 84(Supp. 3): 79-85.

7. Parsons IM, Millett PJ, Warner JJP. Glenoid wear after shoulder hemiarthroplasty. Clin Orthop Relat Res. 2004; 421(421): 120-5.

8. Habermeyer P, Magosch P, Luz V, Lichtenberg S, Lichtenberg AS. Radiographic analysis three-dimensional glenoid deformity in patients with osteoarthritis: a three-dimensional glenoid deformity in patients 
with osteoarthritis: a radiographic analysis. J Bone Joint Surg Am. 2006; 88: 1301-7.

9. Richards RR, An KN, Bigliani LU, Friedman RJ, Gartsman GM, Gristina AG, et al. A standardized method for the assessment of shoulder function. J Shoulder Elbow Surg. 1994; 3(6): 347-52.

10. Constant CR, Murley AH. A clinical method of functional assessment of the shoulder. Vol. NA; Clin Orthop Relat Res. 1987; 214: 160-4.

11. Hervás MT, Navarro CM, Peiró S, Rodrigo PJ, López MP, Martínez TI. Versión Española del cuestionario DASH. Adaptación transcultural, fiabilidad, validez y sensibilidad a los cambios. Med Clin. 2006; 127(12): 441-7.

12. Walch G, Badet R, Boulahia A, Khoury A. Morphologic study of the glenoid in primary glenohumeral osteoarthritis. J Arthroplasty. 1999; 14(6): 756-60.

13. Gartsman GM, Roddey TS, Hammerman SM. Shoulder arthroplasty with or without resurfacing of the glenoid in patients who have osteoarthritis shoulder arthroplasty with or without resurfacing of the glenoid in patients who have osteoarthritis. J Bone Joint Surg Am. 2000; 82(1): 26-34.

14. Gadea F, Alami G, Pape G, Boileau P, Favard L. Shoulder hemiarthroplasty: Outcomes and long-term survival analysis according to etiology. Orthop Traumatol Surg Res. 2012; 98(6): 659-65.

15. Lo IKY, Litchfield RB, Griffin S, Faber K, Patterson SD, Kirkley A. Quality-of-life outcome following hemiarthroplasty or total shoulder arthroplasty in patients with osteoarthritis. A prospective, randomized trial. J Bone Joint Surg Am. 2005; 87(10): 2178-85.

16. Levine WN, Djurasovic M, Glasson JM, Pollock RG, Flatow EL, Bigliani LU. Hemiarthroplasty for glenohumeral osteoarthritis: results correlated to degree of glenoid wear. J Shoulder Elbow Surg. 1997; 6(5): 449-54.

17. Edwards TB, Kadakia NR, Boulahia A, Kempf JF, Boileau P, Némoz C, et al. A comparison of hemiarthroplasty and total shoulder arthroplasty in the treatment of primary glenohumeral osteoarthritis: Results of a multicenter study. J Shoulder Elb Surg. 2003; 12(3): 207-13.

18. Duan X, Zhang W, Dong X, Liu M, Gao Y, Huang F, et al. Total shoulder arthroplasty versus hemiarthroplasty in patients with shoulder osteoarthritis: A meta-analysis of randomized controlled trials. Semin Arthritis Rheum. 2013; 43(3): 297-302.

19. Bryant D, Litchfield R, Sandow M, Gartsman GM, Guyatt G, Kirkley A. A comparison of pain, strength, range of motion, and functional outcomes after hemiarthroplasty and total shoulder arthroplasty in patients with osteoarthritis of the shoulder. A systematic review and meta-analysis. J Bone Joint Surg Am. 2005; 87(9): 1947-56.

20. Carroll RM, Izquierdo R, Vazquez M, Blaine TA, Levine WN, Bigliani LU. Conversion of painful hemiarthroplasty to total shoulder arthroplasty: long-term results. J Shoulder Elbow Surg. 2004; 13(6): 599-603.

21. Orfaly RM, Rockwood CA, Esenyel CZ, Wirth MA. A prospective functional outcome study of shoulder arthroplasty for osteoarthritis with an intact rotator cuff. J Shoulder Elbow Surg. 2003; 12(3): 214-21.

Financiamiento: La presente investigación no ha recibió ayudas específicas provenientes de agencias del sector público, sector comercial o entidades sin ánimo de lucro.

Conflicto de intereses: Ninguno. 